\title{
Room-Temperature Exciton-Polariton Condensation in a Tunable Zero-Dimensional Microcavity
}

\author{
Fabio Scafirimuto, ${ }^{\dagger}$ Darius Urbonas, $^{\dagger}$ Ullrich Scherf, ${ }^{\ddagger}$ Rainer F. Mahrt, ${ }^{\dagger}$ and Thilo Stöferle ${ }^{*}{ }^{\dagger} \odot$ \\ ${ }^{\dagger}$ IBM Research - Zurich, Säumerstrasse 4, 8803 Rüschlikon, Switzerland \\ ${ }^{\ddagger}$ Macromolecular Chemistry Group and Institute for Polymer Technology, Bergische Universität Wuppertal, Gauss-Strasse 20, 42119 \\ Wuppertal, Germany
}

Supporting Information

\begin{abstract}
We create exciton-polaritons in a zero-dimensional (OD) microcavity filled with organic ladder-type conjugated polymer in the strong light-matter interaction regime. Photonic confinement at wavelength scale is realized in the longitudinal direction by two dielectric Bragg mirrors and laterally by a submicron Gaussian-shaped defect. The cavity is separated into two parts, allowing nanometer position control and enabling tuning of the exciton and photon fractions of the polariton wave function. Polariton condensation is achieved with nonresonant picosecond optical excitation under ambient conditions and evidenced by a threshold behavior with a nonlinear increase in the emission intensity, line narrowing,

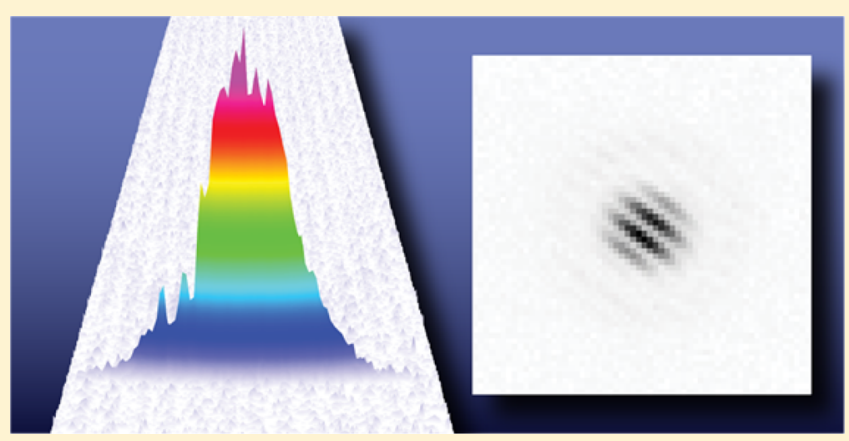
and a blue shift in the emission peak. Furthermore, angular emission spectra show that condensation occurs in the ground state of the $0 \mathrm{D}$ cavity, and first-order coherence measurements reveal the coherent nature. These experiments open the door for polariton quantum fluids in complex external potentials at room temperature.
\end{abstract}

KEYWORDS: polariton condensate, tunable microcavity, organic polymer, zero-dimensional, strong coupling

巨 xciton-polaritons are light-matter excitations generated by the coupling of photons inside a high-quality-factor optical resonator with an embedded optically active material. By matching the energy of the optical resonance (cavity photons) and the material's optoelectronic resonance transition (excitons, i.e., bound electron-hole pairs) it is possible to observe the characteristic anticrossing behavior that reflects the emergence of new eigenstates. ${ }^{1}$ These exciton-polaritons are quasi-particles of bosonic nature. At sufficiently high density, they can undergo nonequilibrium Bose-Einstein condensation and form a polariton condensate. ${ }^{2,3}$ This macroscopic quantum phenomenon has been observed in various inorganic semiconductors ${ }^{4}$ and more recently in organic materials, ${ }^{,-7}$ even up to room temperature, which is very appealing in view of future possible technological applications.

Motivated by the success of ultracold atomic gases, ${ }^{10}$ the use of a condensate for analog quantum simulations of solid-state and nonequilibrium toy-model Hamiltonians ${ }^{11}$ has recently received enormous attention. Here it is crucial to be able to create arbitrary potential landscapes for the quantum fluid to emulate different kinds of crystal lattices and the intricate effects that arise from the various band structures, e.g., the Dirac cones in graphene. Furthermore, through strong lateral confinement, lower-dimensional geometries like one-dimensional nanowires can be simulated. Lithographic definition of small mesas, ${ }^{12}$ deep etching of the whole microcavity structure, $^{13}$ and patterning of thin metal layers ${ }^{14}$ have been used to realize confinement for semiconductor microcavities. Wavelength tunability can be achieved through separating the two cavity mirrors and mounting them on nanopositioners. ${ }^{15}$ Gaussian-shaped nanoscale defect structures are enabling much tighter, wavelength-scale lateral confinement ${ }^{16}$ with theoretical cavity quality factors above $10^{5}$ that can be fabricated using focused ion beam (FIB) milling. ${ }^{17}$

In this work, we report the observation of room-temperature polariton condensation in a tunable Gaussian-shaped microcavity based on an organic polymer. The material used is a methyl-substituted ladder-type poly( $p$-phenylene) (MeLPPP) ${ }^{18}$ which is a $\pi$-conjugated organic semiconductor polymer with a comparably rigid backbone that has already been used to show polariton condensation in flat Fabry-Pérot cavities. ${ }^{5}$ We build on a configuration described by Urbonas et al. ${ }^{19}$ enabling tunable room-temperature strong coupling. It consists of two cavity halves that are mounted on XYZ nanopositioners (Figure 1a; see Methods for details). One part consists of a glass mesa with a Gaussian defect (Figure 1b) and a subsequently deposited distributed Bragg reflector (DBR). The other one consists of a DBR and spin-coated thin film of

Special Issue: Strong Coupling of Molecules to Cavities

Received: May 31, 2017

Published: September 29, 2017 

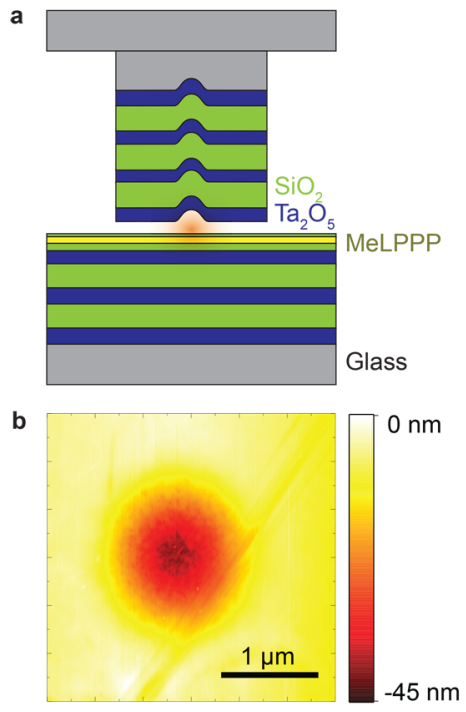

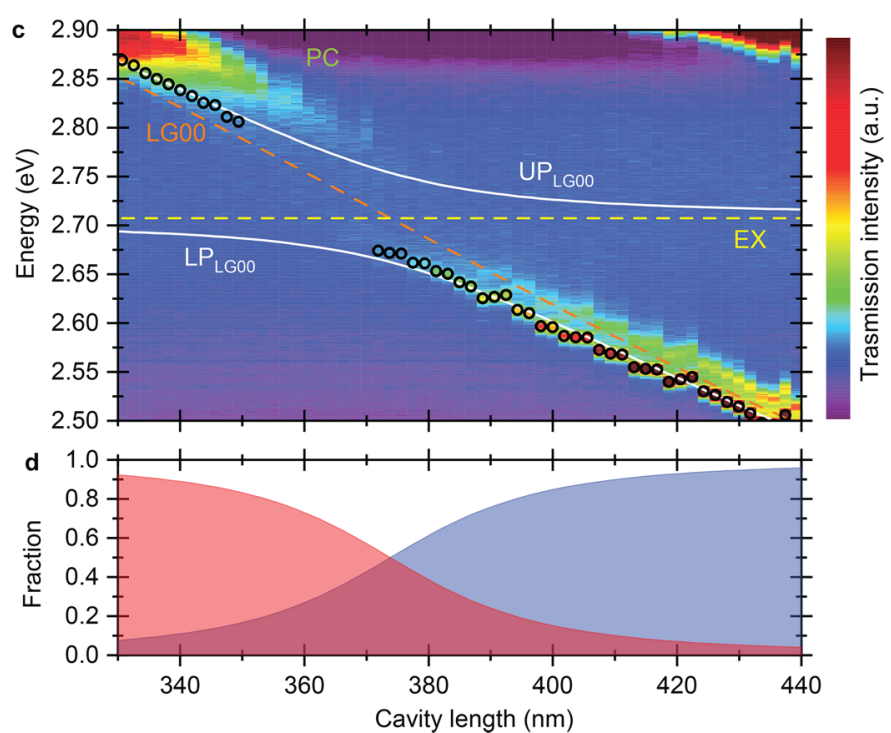

Figure 1. (a) Sketch of the tunable Gaussian defect cavity. (b) Atomic force microscopy image of the Gaussian defect structure. (c) Transmission spectrum as a function of distance between the cavity halves, showing the avoided crossing of the localized mode from the Gaussian defect (LG00, orange dashed line) and the exciton of the MeLPPP (EX, yellow dashed line) into lower $\left(\mathrm{LP}_{\mathrm{LG00}}\right)$ and upper $\left(\mathrm{UP}_{\mathrm{LG00}}\right)$ polariton branches. The black circles are extracted transmission peak centers from the measurement, whereas the solid white lines are fits to these data points with a coupled oscillators model. (d) Composition of the polariton wave function of the $\mathrm{LP}_{\mathrm{LG} 00}$ branch (photonic $=$ blue, excitonic $=$ red) calculated from the Hopfield coefficients as the cavity length is tuned.

MeLPPP (see the Supporting Information for details) protected by a thin inorganic layer.

Compared with an unpatterned cavity, the Gaussian defect leads to zero-dimensional (OD) localization of the optical mode, but this transverse confinement does not change the cavity $Q$ apart from additional losses induced by the FIB fabrication process. ${ }^{17}$ The experimental resonance line width, including the absorption from the polymer layer, corresponds to $Q \sim 600$ (measured at $95 \%$ photon fraction in luminescence). The configuration consisting of separated cavity halves provides control over the distance between the mirrors and hence allows the composition of the polariton wave function to be varied between more photon-like and more exciton-like. To observe the strong-coupling regime manifested by the splitting of the cavity mode into upper and lower polariton branches, we illuminate the sample with a broadband halogen lamp and record the transmission spectrum while tuning the cavity resonance across the exciton energy (Figure 1c). In addition to the lowest Laguerre-Gaussian mode (LG00) from the cylindrically symmetric OD cavity, we also observe the planar cavity mode (PC) because our probe beam is larger than the Gaussian defect. By fitting the upper and lower polariton branches of the LG00 mode with a coupled oscillators model, we extract a Rabi splitting of $2 \Omega=92 \mathrm{meV}$. The change in the wave function composition as the cavity resonance is tuned can be inferred by calculating the Hopfield coefficients (Figure 1d). The transmission peak full-width at half-maximum (fwhm) of the lower polariton branch is $8 \mathrm{meV}$ at $90 \%$ photon fraction and $22 \mathrm{meV}$ at $50 \%$ photon fraction.

To achieve polariton condensation, we excite the microcavity off-resonantly at $3.1 \mathrm{eV}$ photon energy under normal incidence, outside the DBR band gap. We use a single-mode photonic crystal fiber to obtain a small excitation spot close to the dimensions of the Gaussian defect and to stretch the excitation laser pulse to $\sim 12$ ps duration. The emitted light is either detected by a camera (real-space image) or a spectrograph equipped with a two-dimensional detector or is fed into a
Michelson interferometer (see Methods for details). Above a certain threshold we find nonlinear behavior in the total emitted intensity, a narrowing of the line width of the polariton mode, and a blue shift of the peak position. Upon comparison of the angular emission patterns below and above threshold, a dramatic increase in the polariton population in the lowestenergy mode is observed. Finally, first-order coherence measurements allow estimation of the temporal coherence length.

\section{RESULTS}

The $0 \mathrm{D}$ character of the microcavity has a pronounced impact on the angular emission spectrum. While the PC mode has a parabolic dispersion relation, the Laguerre-Gaussian modes LG $n l$ (where $n$ and $l$ give the numbers of nodes in the radial and azimuthal directions, respectively) have discrete energies. At low excitation power (Figure 2a), we observe the lowestazimuthal-order mode (LG00) and the first-order mode (LG01) from the Gaussian defect and the PC mode from the area pumped in the vicinity of the Gaussian defect. At high excitation power (Figure $2 \mathrm{~b}$ ), the situation changes completely: the polaritons condense in the LG00 mode (lowest-energy mode) while the other modes are so weakly populated that they are barely observable. Already from this measurement the nonlinear increase in the emission, the line narrowing, and the blue shift, all of which are indications of polariton condensation, are visible. Furthermore, this shows that condensation in the lowest-energy state indeed occurs. Polariton lasing ${ }^{12}$ from the higher LG01 mode was observed only occasionally when the cavity was detuned to an extremely photonic polariton composition (>97\% photon contribution).

To obtain quantitatively the threshold excitation density $P_{\text {th }}$ for the condensation, we measured the angularly and spatially integrated emission spectrum versus the excitation fluence. The recorded spectra of the lowest polariton branch of the LG00 mode were fitted with a Gaussian peak, allowing its area, width, and center to be extracted. The low emission intensity far 


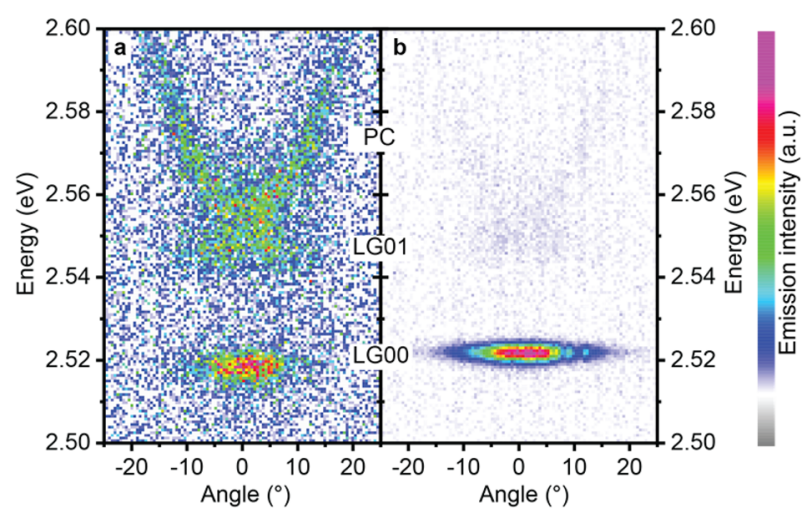

Figure 2. Angular dispersion of the emitted light measured with an imaging spectrograph in the quasi-momentum-space configuration. The color scale is normalized to the intensity maximum in each plot, and the exposure times were $60 \mathrm{~s}$ for the left graph and $10 \mathrm{~s}$ for the right graph. (a) At an excitation fluence below threshold $\left(0.5 P_{\text {th }}\right)$, the parabolic dispersion of the planar cavity (PC) mode and the flat bands of the LG00 and higher-order LG01 modes are observed. (b) Above threshold $\left(2 P_{\text {th }}\right)$, the mode structure is similar, but the intensity distribution is completely changed. While the LG01 and PC modes are barely visible, the lowest-energy mode, LG00, is strongly increased because of the stimulated scattering into the condensate. It is notable that all of the modes exhibit a slight blue shift due the polaritonexciton interaction and saturation effects.

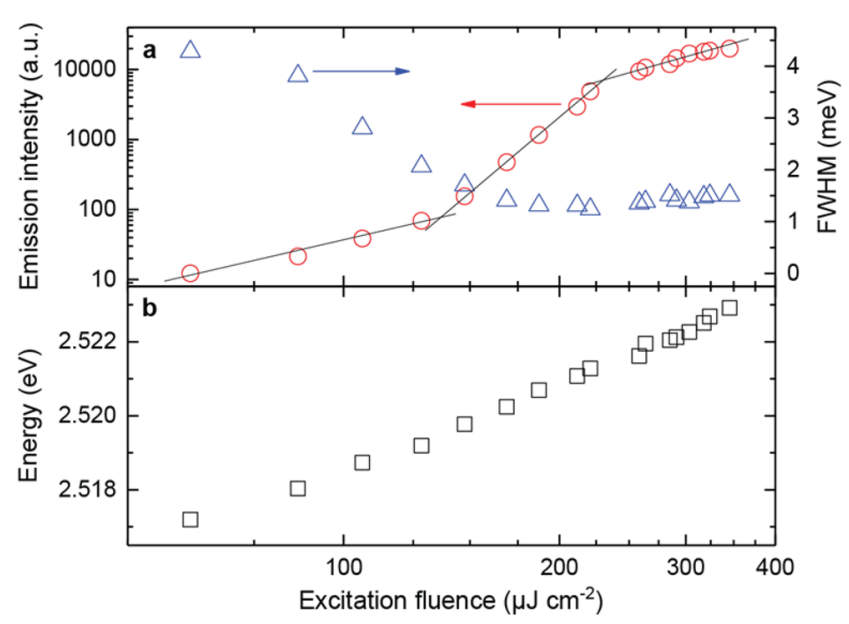

Figure 3. (a) Plot of emission intensity vs excitation fluence (red open circles, left axis, log-log scale) together with the emission line width (blue open triangles, right axis). A threshold for the superlinear increase is observed at around $130 \mu \mathrm{J} \mathrm{cm}^{-2}$ (lines are guides to the eye). This coincides with a decrease in the line width from $\sim 4.5 \mathrm{meV}$ below threshold to $\sim 1.5 \mathrm{meV}$ above threshold. (b) Power-dependent blue shift in the polariton emission.

below the threshold limits the range where the fit works reliably to as low as $\sim 0.5 P_{\text {th }}$. In Figure 3 a, the plot of total emitted intensity (area of the peak) versus excitation power displays the characteristic nonlinear behavior due to the stimulated scattering into the polariton ground state. $P_{\text {th }}$ is $130 \mu \mathrm{J} \mathrm{cm}^{-2}$, which is almost 4 times lower than the value for the same material stack obtained in an unpatterned, nontunable cavity with a slightly higher excitonic wave function fraction. ${ }^{5}$ Furthermore, the peak width exhibits a significant drop of the line width, corresponding to transition toward the condensate regime. For some samples, we found peak widths down to 300 $\mu \mathrm{eV}$ (see the Supporting Information), which is almost 1 order of magnitude narrower than for planar cavities ${ }^{5}$ and corresponds to the Fourier limit imposed by the pump duration. This points toward different condensation dynamics in the OD cavity, where only one discrete lowest state exists and which is essentially free of photonic spatial disorder and potential influence of instabilities of the polariton reservoir. ${ }^{20}$

The peak center energy as displayed in Figure $3 \mathrm{~b}$ shows the typical blue shift due to the interaction among the polaritons and the exciton reservoir ${ }^{21}$ and saturation effects. ${ }^{20}$ In the range of excitation powers and polariton energies studied, the behavior is well-described by a linear to sublinear increase in the energetic shift with excitation power (power law with exponent between 0.3 and 1.0) and does not exhibit a pronounced jump when the condensate regime is entered. For a OD cavity mode with flat dispersion, it is not possible to access the effective refractive index independent from the blue shift. However, from the curvature of the parabolic dispersion curve in a planar cavity with the same material and similar light-matter coupling strength, it is known ${ }^{5}$ that no transition to the weak-coupling regime occurs when the excitation density is increased above the condensation threshold. It must be noted that the variability of these quantities (threshold, peak width, blue shift) for different spots of the conjugated polymer can be quite high. For example, if the same Gaussian defect structure is tuned to the same resonance energy (i.e., the same wave function composition) by adjusting the distance on different lateral positions of the lower cavity half, the threshold fluence can vary up to $50 \%$.

Finally, we study the first-order coherence of the condensate by coupling the polariton emission into a Michelson interferometer. The emission is split and coupled into two interferometer arms, where one can be delayed and is spatially inverted with respect to the other, and then both real-space images are focused on a camera. As the light originates from the Gaussian-shaped LG00 mode, the interferogram is a Gaussian with superimposed fringes (Figure 4a), and the fringe period corresponds to the relative angle under which the beams were overlapped on the camera. We obtain the temporal coherence from the fringe amplitude as a function of interferometer arm delay by Fourier transformation of the images (Figure $4 \mathrm{~b}$ ). Below threshold, the polariton emission has a very short coherence time, and we find a very short decay of the coherence within 10 fs. Above threshold, the polariton condensate gives rise to dramatically prolonged coherence by about 2 orders of magnitude, up to a few picoseconds. For some spatial positions on the polymer, the coherence above threshold shows exponential falloff characteristics and for others Gaussian falloff characteristics, ${ }^{22}$ which can point toward different magnitudes of fluctuations dependent on the local excitonic disorder. ${ }^{23}$

\section{CONCLUSIONS}

We have demonstrated exciton-polariton condensation in a $\mathrm{OD}$ microcavity under ambient conditions, as evidenced by a comprehensive set of experimental results. The submicronsized tunable defect structure establishes a promising platform for the investigation of quantum fluids in arbitrary potential landscapes for analog quantum simulations.

\section{METHODS}

Sample Preparation. To realize the top part of the cavity, we first defined a mesa structure $(\sim 200 \mu \mathrm{m}$ diameter and $\sim 30$ 

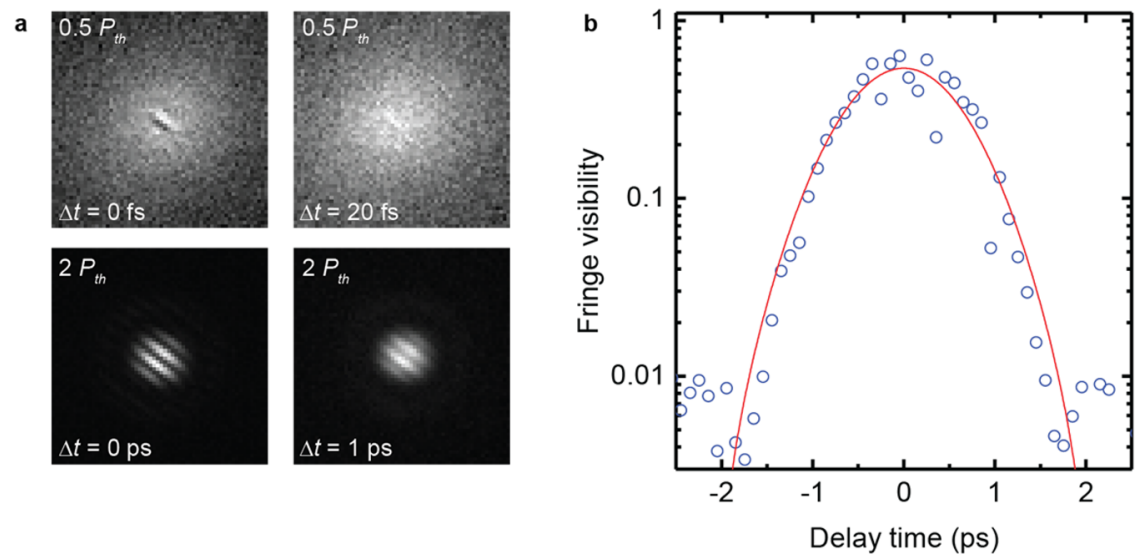

Figure 4. Fringe visibility of the polariton emission as a function of relative delay time $\Delta t$ between the Michelson interferometer arms. (a) Exemplary recorded real-space images (dimensions $6.6 \mu \mathrm{m} \times 6.6 \mu \mathrm{m}$, grayscale normalized for each image). Below threshold, the coherence lasts only about 10 fs. Above threshold, the polariton condensate is formed, resulting in a perfect Gaussian envelope with a $1 / \mathrm{e}^{2}$ radius of $450 \mathrm{~nm}$, corresponding to the spatial extension of the LG00 mode. The coherence is extended by 2 orders of magnitude up to more than a picosecond. The imaging exposure times are $30 \mathrm{~s}$ for the data set below threshold and $1 \mathrm{~s}$ above threshold. (b) For quantitative analysis of the condensate temporal coherence, the fringe visibility was extracted from the Fourier transform of the images by using the magnitude at the fringes' spatial period. The solid line shows a Gaussian fit to the data. The fringe visibility is normalized to the value deduced directly from the raw images at zero time delay, giving maximum fringe amplitudes of $60-70 \%$ for both data sets below and above threshold.

$\mu \mathrm{m}$ height) by back-etching on a borosilicate substrate, and then we patterned the Gaussian defects (depth $\sim 30 \mathrm{~nm}$ and fwhm $\sim 1000 \mathrm{~nm})$ by focused ion beam milling. Finally, we deposited 6.5 double layers of $\mathrm{Ta}_{2} \mathrm{O}_{5} / \mathrm{SiO}_{2}$ forming the $\mathrm{DBR}$ by magnetron sputtering. The bottom part was realized by sputtering 9.5 double layers on a borosilicate substrate. Subsequently, we deposited a $35 \mathrm{~nm}$ thin layer of the MeLPPP polymer by spin-coating from a toluene solution ( $1 \%$ concentration) and completed the structure by sputtering a $10 \mathrm{~nm}$ protective layer of $\mathrm{SiO}_{2}$ on top. This protective layer increased the photostability above threshold by about an order magnitude to $>3 \times 10^{5}$ excitation laser shots compared with an unprotected MeLPPP film, and the encapsulation could be even further improved, e.g., with an atomic-layer-deposited $\mathrm{Al}_{2} \mathrm{O}_{3}$ film to support $>10^{6}$ shots.

Optical Characterization. The two cavity parts were mounted on independent $X Y Z$ stages that allowed nanometer control of the positions. The microcavity was excited either with a multimode fiber-coupled halogen lamp for the transmission measurements or with a single-mode fiber-coupled ( $2 \mathrm{~m}$ polarization maintaining photonic crystal fiber, $5 \mu \mathrm{m}$ mode diameter) frequency-doubled regenerative amplifier seeded by a mode-locked Ti:sapphire laser for all other measurements. The initial pump pulse was $100-200$ fs with a repetition rate of $1 \mathrm{kHz}$. The pump intensity at the fiber output was controlled with a movable gradient filter. The excitation beam was focused through the top cavity part with a longworking-distance apochromatic microscope objective (100X, numerical aperture $(\mathrm{NA})=0.5$ ) to a spot with fwhm $=2-3 \mu \mathrm{m}$ on the sample. The emitted light from the cavity was collected through the bottom part with an apochromatic microscope objective $(20 \times, \mathrm{NA}=0.5)$ that was mounted on a third $\mathrm{XYZ}$ nanopositiong stage. Suitable long-pass filters were used to block the excitation light. The emission was detected either by an imaging spectrograph (liquid $\mathrm{N}_{2}$-cooled charge-coupled device (CCD), $0.5 \mathrm{~m}$ focal length, 1800 lines/mm grating for the spectra and 300 lines $/ \mathrm{mm}$ for the dispersion curves) or by a cooled CCD camera (for the real-space images). For the dispersion curves, the spectrograph performed $k$-space imaging with an entrance slit width of $50 \mu \mathrm{m}$. For the coherence measurements, the emitted light was split by a nonpolarizing beamsplitter cube and recombined and focused after the Michelson interferometer on a cooled CCD camera. In one interferometer arm, a hollow retroreflector (used to invert the image) was mounted, and a motorized linear stage and an additional piezo provided the variable time delay.

\section{ASSOCIATED CONTENT}

\section{S Supporting Information}

The Supporting Information is available free of charge on the ACS Publications website at DOI: 10.1021/acsphotonics.7b00557.

Additional characterization of the polymer layer and data on narrow-line-width condensates (PDF)

\section{AUTHOR INFORMATION}

\section{Corresponding Author}

*E-mail: tof@zurich.ibm.com.

ORCID

Thilo Stöferle: 0000-0003-0612-7195

\section{Author Contributions}

The manuscript was written through contributions of all authors. All authors have given approval to the final version of the manuscript.

\section{Notes}

The authors declare no competing financial interest.

\section{ACKNOWLEDGMENTS}

We are grateful to W. Riess, G.-L. Bona, and A. Imamoglu for discussions and U. Drechsler, R. Stutz, S. Reidt, and M. Sousa for help with the sample preparation and characterization. This work was partly supported by the Swiss State Secretariat for Education, Research and Innovation (SERI) and the European Union's Horizon-2020 Framework Program through the MarieSklodowska Curie ITN Networks PHONSI (H2020-MSCAITN-642656) and SYNCHRONICS (H2020-MSCA-ITN643238). 


\section{REFERENCES}

(1) Weisbuch, C.; Nishioka, M.; Ishikawa, A.; Arakawa, Y. Observation of the Coupled Exciton-Photon Mode Splitting in a Semiconductor Quantum Microcavity. Phys. Rev. Lett. 1992, 69, 33143317.

(2) Kasprzak, J.; Richard, M.; Kundermann, S.; Baas, A.; Jeambrun, P.; Keeling, J. M. J.; Marchetti, F. M.; Szymańska, M. H.; André, R.; Staehli, J. L.; Savona, V.; Littlewood, P. B.; Deveaud, B.; Dang, L. S. Bose-Einstein Condensation of Exciton Polaritons. Nature 2006, 443, 409-414.

(3) Carusotto, I.; Ciuti, C. Quantum Fluids of Light. Rev. Mod. Phys. 2013, 85, 299-366.

(4) Deng, H.; Haug, H.; Yamamoto, Y. Exciton-Polariton BoseEinstein Condensation. Rev. Mod. Phys. 2010, 82, 1489-1537.

(5) Plumhof, J. D.; Stöferle, T.; Mai, L.; Scherf, U.; Mahrt, R. RoomTemperature Bose-Einstein Condensation of Cavity Exciton-Polaritons in a Polymer. Nat. Mater. 2014, 13, 247-252.

(6) Daskalakis, K. S.; Maier, S. A.; Murray, R.; Kéna-Cohen, S. Nonlinear Interactions in an Organic Polariton Condensate. Nat. Mater. 2014, 13, 271-278.

(7) Dietrich, C. P.; Steude, A.; Tropf, L.; Schubert, M.; Kronenberg, N. M.; Ostermann, K.; Höfling, S.; Gather, M. C. Sci. Adv. 2016, 2, e1600666.

(8) Lagoudakis, P. Polariton Condensates: Going Soft. Nat. Mater. 2014, 13, 227-228.

(9) Sanvitto, D.; Kéna-Cohen, S. The Road Towards Polaritonic Devices. Nat. Mater. 2016, 15, 1061-1073.

(10) Bloch, I.; Dalibard, J.; Nascimbène, S. Quantum Simulations with Ultracold Quantum Gases. Nat. Phys. 2012, 8, 267-276.

(11) Kim, N. Y.; Yamamoto, Y. Exciton-Polariton Quantum Simulators. In Quantum Simulations with Photons and Polaritons; Angelakis, D. G., Ed.; Springer International Publishing: Cham, Switzerland, 2017; pp 91-121.

(12) Kaitouni, R. I.; El Daif, O.; Baas, A.; Richard, M.; Paraiso, T.; Lugan, P.; Guillet, T.; Morier-Genoud, F.; Ganière, J. D.; Staehli, J. L.; Savona, V.; Deveaud, B. Engineering the Spatial Confinement of Exciton Polaritons in Semiconductors. Phys. Rev. B: Condens. Matter Mater. Phys. 2006, 74, 155311.

(13) Bajoni, D.; Senellart, P.; Wertz, E.; Sagnes, I.; Miard, A.; Lemaïtre, A.; Bloch, J. Polariton Laser Using Single Micropillar GaAsGaAlAs Semiconductor Cavities. Phys. Rev. Lett. 2008, 100, 047401.

(14) Lai, C. W.; Kim, N. Y.; Utsunomiya, S.; Roumpos, G.; Deng, H.; Fraser, M. D.; Byrnes, T.; Recher, P.; Kumada, N.; Fujisawa, T.; Yamamoto, Y. Coherent Zero-State and pi-State in an ExcitonPolariton Condensate Array. Nature 2007, 450, 529.

(15) Prakash, G. V.; Besombes, L.; Kelf, T.; Baumberg, J. J.; Bartlett, P. N.; Abdelsalam, M. E. Tunable Resonant Optical Microcavities by Self-Assembled Templating. Opt. Lett. 2004, 29, 1500-1502.

(16) Ding, F.; Stöferle, T.; Mai, L.; Knoll, A.; Mahrt, R. F. Vertical Microcavities with High $Q$ and Strong Lateral Mode Confinement. Phys. Rev. B: Condens. Matter Mater. Phys. 2013, 87, 161116.

(17) Mai, L.; Ding, F.; Stöferle, T.; Knoll, A.; Offrein, B. J.; Mahrt, R. F. Integrated Vertical Microcavity Using a Nano-Scale Deformation for Strong Lateral Confinement. Appl. Phys. Lett. 2013, 103, 243305.

(18) Scherf, U.; Bohnen, A.; Müllen, K. Polyarylenes and poly(arylenevinylene)s,9 The Oxidized States of a (1,4-phenylene) Ladder Polymer. Makromol. Chem. 1992, 193, 1127-1133.

(19) Urbonas, D.; Stöferle, T.; Scafirimuto, F.; Scherf, U.; Mahrt, R. F. Zero-Dimensional Organic Exciton-Polaritons in Tunable Coupled Gaussian Defect Microcavities at Room Temperature. ACS Photonics 2016, 3, 1542-1545.

(20) Bobrovska, N.; Matuszewski, M.; Daskalakis, K. S.; Maier, S. A.; Kéna-Cohen, S. Dynamical Instability of a Nonequilibrium ExcitonPolariton Condensate. ACS Photonics, DOI: 10.1021/acsphotonics.7b00283.

(21) Stöferle, T.; Plumhof, J. D.; Mai, L.; Scherf, U.; Mahrt, R. F. Exciton-Polariton Bose-Einstein Condensation with a Polymer at Room Temperature. Proc. SPIE 2015, 9370, 93702T.
(22) Love, A. P. D.; Krizhanovskii, D. N.; Whittaker, D. M.; Bouchekioua, R.; Sanvitto, D.; Al Rizeiqi, S.; Bradley, R.; Skolnick, M. S.; Eastham, P. R.; André, R.; Dang, L. S. Le Si Dang. Intrinsic Decoherence Mechanisms in the Microcavity Polariton Condensate. Phys. Rev. Lett. 2008, 101, 067404.

(23) Whittaker, D. M.; Eastham, P. R. Coherence Properties of the Microcavity Polariton Condensate. EPL 2009, 87, 27002. 